\title{
À propos de graines et de métabolisme synthétique
}

Travis Bayer

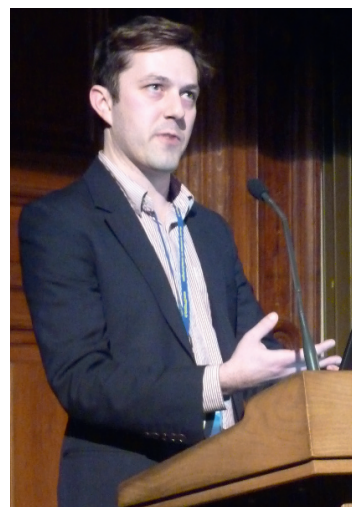

Division of molecular biosciences and Centre for synthetic biology and innovation (CSynBI), Imperial College London, South Kensington Campus, London SW7 2AZ, Royaume-Uni. t.bayer@imperial.ac.uk

Mon laboratoire appartient au Centre for synthetic biology and innovation (CSynBI) à l'Imperial College de Londres. Nous collaborons avec d'autres groupes de scientifiques de notre établissement, ainsi qu'avec des représentants des sciences sociales, Nikolas Rose et Claire Marris, du King's College. Nous sommes également membres d'un consortium britannique, le Flowers Consortium, auquel appartiennent des groupes des universités de Cambridge, Edinburgh et Newcastle.

Mon groupe compte une demi-douzaine de doctorants et autant de post-doctorants issus d'un large horizon de disciplines : microbiologistes, chimistes, chimistes organiciens, physiciens et ingénieurs. Notre ambition est de concevoir des systèmes biologiques comme des unités de fabrication à l'échelle nanoscopique ou macroscopique. Nous voulons utiliser la biologie pour produire une large gamme de composés utiles, de la chimie fine à la pharmacie en passant par les carburants liquides.

\section{Transfert de l'innovation : importance des sciences sociales dans le choix du modèle}

Nous sommes partis des modes opératoires de l'industrie pétrochimique d'aujourd'hui : celle-ci utilise le carbone issu d'une grande variété de sources fossiles - pétrole, charbon ou gaz naturel - et le transforme en

Conférence en anglais - Retranscription et traduction simultanée assurées par Ubiqus. précieux composés que nous utilisons quotidiennement. Pour ce faire, elle recourt à des catalyseurs développés après des siècles de progrès en chimie et en génie des procédés. La biologie suit un parcours comparable: nous tentons de dépasser le stade des dérivés carbonés pour envisager un avenir à faible carbone. Pour ce faire, nous pouvons utiliser des sources de carbone renouvelables, comme le dioxyde de carbone contenu dans l'air, les cultures céréalières ou les déchets ligno-cellulosiques, et les faire transformer en composés et matériaux utiles par des systèmes biologiques modifiés.

Mon groupe travaille sur le maillon central de la chaîne, les systèmes biologiques produits par des usines cellulaires. Nos objectifs scientifiques à court terme sont extrêmement pratiques. Mais, hors du champ strictement scientifique, nous nous intéressons également à la stratégie de transfert de l'innovation hors du laboratoire. Notre interaction avec les chercheurs en sciences sociales de CSynBI est très précieuse à cet égard. Dans cette version 1.0 de notre stratégie de transfert d'innovation, il y a trois étapes successives: le projet de recherche fondamentale réalisé dans un laboratoire académique, la recherche appliquée - qui peut être soutenue ou financée par des partenaires industriels -, enfin il faut convaincre des industriels ou des actionnaires que le projet mérite d'être poursuivi et financé. Le rôle des sciences sociales serait alors de convaincre un public réticent ou l'industrie de participer à cette innovation.

Nos collègues des sciences sociales nous ont montré que ce modèle linéaire comporte d'importantes limites. Celles-ci sont exacerbées par le fait que ce modèle doit, à un moment donné, s'intégrer dans des schémas réglementaires gouvernementaux dont je reparlerai. Nous travaillons donc sur un modèle plus adaptatif ou plus flexible : au lieu 


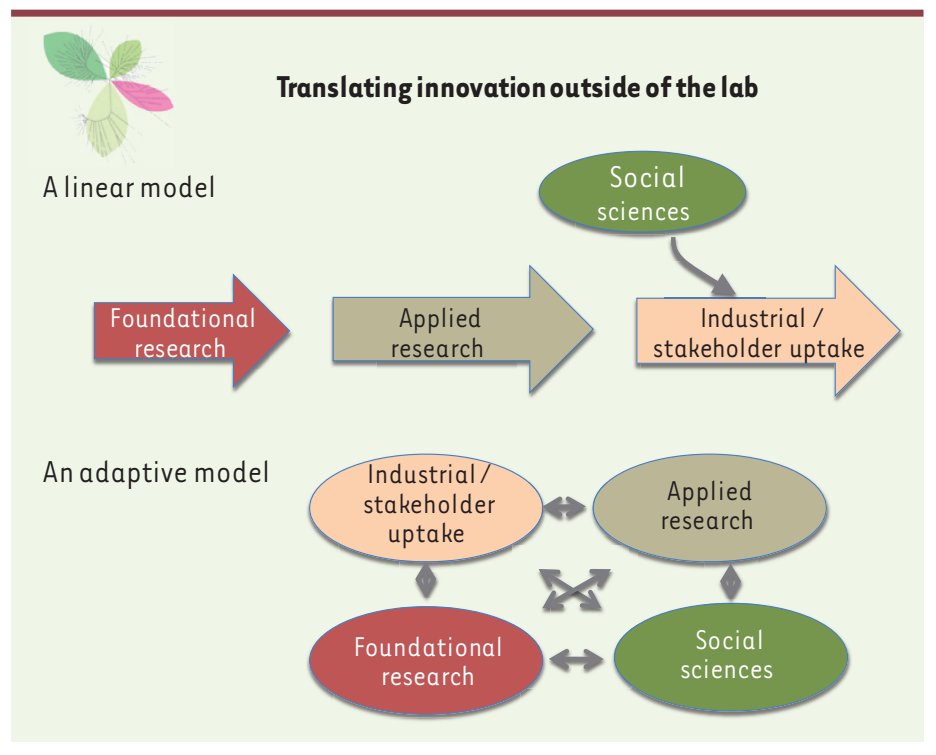

Figure 1. L'objectif du groupe de Travis Bayer est d'associer tous les acteurs dès le démarrage des projets de recherche.

de tabler sur un cheminement linéaire de la recherche fondamentale à l'appropriation par les parties prenantes, nous envisageons d'associer les groupes industriels et les parties prenantes dès le début, ce qui a des répercussions sur la recherche fondamentale, la recherche appliquée, et de déterminer comment les sciences sociales peuvent nous aider à le mettre en place. II ne s'agit plus d'un parcours linéaire, mais d'un dialogue entre tous ces acteurs qui permettra de faire émerger l'innovation à cette interface (Figure 1).

\section{Exemple : la lutte contre le striga, herbe parasite des cultures africaines}

Je prendrai l'exemple de l'un des projets sur lesquels nous travaillons. II porte sur le striga, une herbe parasite qui affecte le riz, le maïs, le sorgho et la cornille, c'est-à-dire des cultures de base, en particulier en Afrique (Figure 2). C'est l'une des principales limites aux rendements agricoles dans les zones tropicales. Ses graines peuvent survivre dans le sol pendant des générations : il est très difficile de s'en débarrasser. Nous nous sommes intéressés à cette plante sur la suggestion de l'un de nos post-doctorants, qui a grandi dans une ferme au Zimbabwe. Certains d'entre nous se sont rendus au Zimbabwe cet été pour échanger avec des agriculteurs et d'autres personnes affectées par le problème. Ces dernières sont essentiellement de petits paysans qui n'ont pas les moyens d'acheter des traitements phytosanitaires.

Sur le plan biologique, les graines de striga restent en dormance dans le sol jusqu'à ce qu'elles détectent une hormone exsudée par une plante hôte, la strigolactone (Figure 2B). Celle-ci entraîne la germination de la graine; le striga se fixe sur l'hôte dont elle absorbe l'humidité et les nutriments et qu'elle empêche souvent de développer une partie aérienne (Figure 2C). Plusieurs stratégies de contrôle sont possibles. La première consiste à développer des cultures résistantes au striga, mais n'a guère été couronnée de succès. La deuxième utilise des semences de céréales résistantes aux herbicides et les enrobe d'herbicides qui affectent les graines de striga quand elles commencent à pousser. L'application de ces deux approches se heurte à des difficultés considérables dans une agriculture vivrière : un petit paysan n'a pas les moyens de les mettre en œuvre. Une troisième piste consiste à éliminer le striga grâce à la germination « suicide ». La germination des graines de striga est en effet conditionnée par la strigolactone émanant des racines de la plante hôte. Le radicule du striga s'étend vers la plante et s'y fixe au moyen d'un haustorium (Figure 2C). II doit impérativement établir le contact sous deux à trois jours sous peine de dégénérescence. Il est donc possible d'induire une germination « suicide » des graines de striga par une substance ressemblant à la strigolactone, et ce avant de semer les plantes hôtes. Mais de tels composés sont très coûteux à produire par les procédés chimiques : une entreprise belge les vend 850 euros les $20 \mathrm{mg}$, ce qui porte le traitement d'un champ entier à des centaines de milliers d'euros, voire des millions. Cette option n'est donc pas réaliste.

Notre projet vise à concevoir une levure capable de fabriquer un composé analogue à la strigolactone ; la fabrication de cette substance deviendrait aussi aisée que de brasser de la bière. Nous connaissons d'ores et déjà les gènes qu'il est nécessaire d'intégrer à la levure pour qu'elle produise la strigolactone ou des composés semblables, même si cette voie de biosynthèse n'a été étudiée que récemment et n'a pas été complétement élucidée : de nombreux projets de recherche fondamentale se consacrent actuellement à cette question. Nous pouvons nous appuyer sur les travaux de recherche fondamentale effectués sur la plante modèle Arabidopsis, car il existe des mutants de cette plante dont les racines ne produisent pas de strigolactone et qui n'y sont pas sensibles. Nous devons enfin encore trouver les étapes de catalyse spécifiques.

Le système que nous avons conçu suit une voie métabolique qui part d'un métabolite essentiel de la levure pour aboutir à un composé analogue à la strigolactone. La première étape consiste à concevoir une levure capable de fabriquer le lycopène, que vous connaissez probablement comme supplément alimentaire et que I'on trouve notamment dans les tomates. La première étape implique de modifier génétiquement la levure par l'ajout de trois gènes qui permettent de transformer le métabolite essentiel de la levure en lycopène. La deuxième étape va du lycopène au $\beta$-carotène et jusqu'à la strigolactone ; elle requiert cinq gènes supplémentaires 


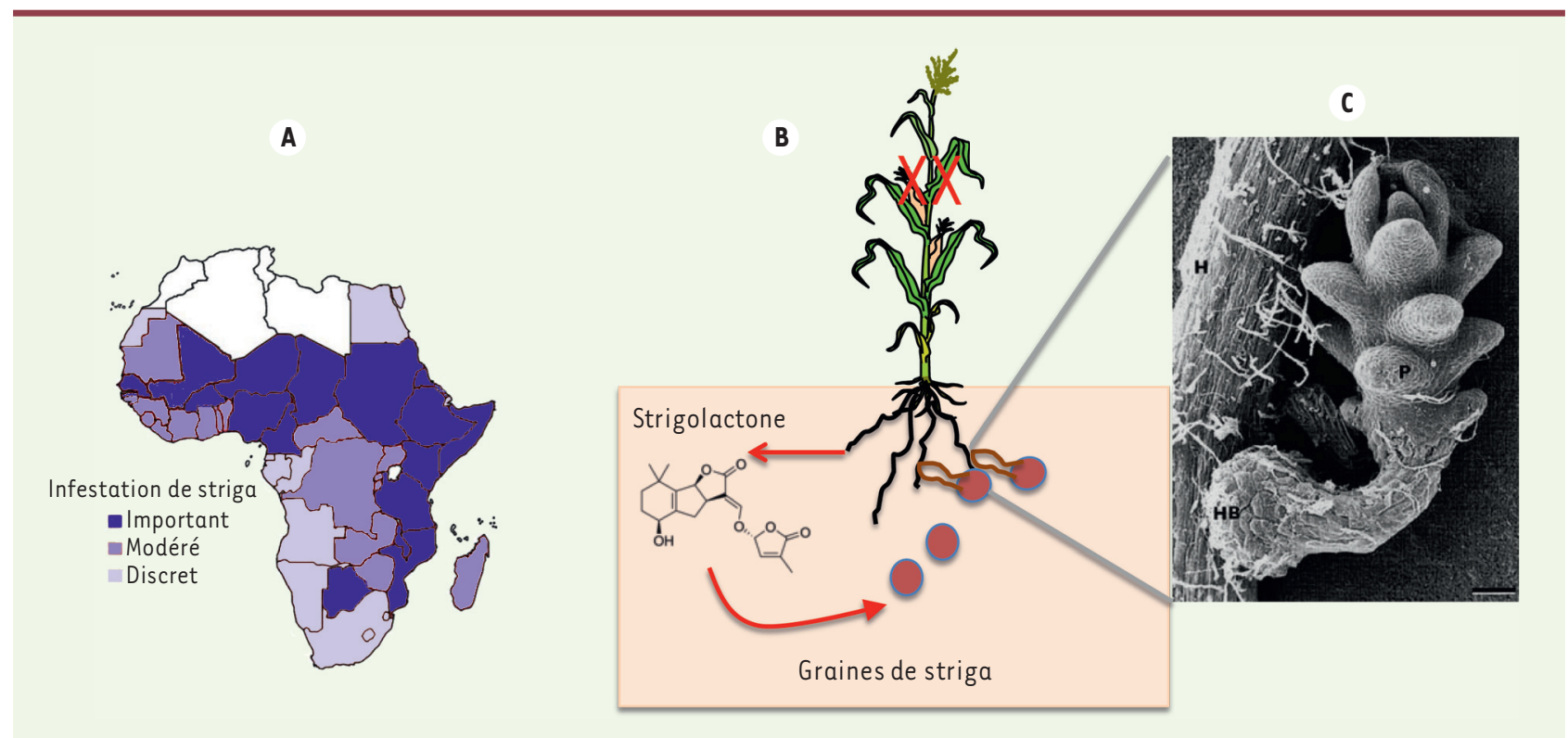

Figure 2. Le problème posé par le striga, une herbe parasite des cultures. A. Dissémination du parasite striga en Afrique (adapté de [1]). B. Schéma du mécanisme d'action du striga. C. Image obtenue par microscopie à balayage de la fixation sur une racine hôte (H) d'une graine de striga après germination (P) (๔ image reproduite de [2] avec la permission de Oxford University Press).

issus d'Arabidopsis et d'Oryza sativa (riz asiatique), et optimisés pour s'exprimer efficacement dans les cellules de levure.

Le coût total de la synthèse et de l'assemblage de ces constructions géniques est de moins de 15000 dollars : le coût de la synthèse génique a considérablement diminué depuis les travaux de Jay Keasling sur l'artémisinine. Nous avons également pu obtenir les séquences génétiques en moins de deux semaines et les assembler en moins d'une semaine. Enfin, leur insertion dans la levure a permis la synthèse d'un composé très proche de la strigolactone.

II restait à vérifier que le composé était effectivement capable de provoquer la germination de striga. Nous avons collecté sur une terre agricole au Zimbabwe des graines de striga, que nous avons cultivées en présence du germinatif produit par la levure ; nous avons compté les radicules émergentes (celles-ci croissent en deux jours). Là où le composé synthétique commercial évoqué précédemment entraîne un taux de germination de $12 \%$, notre composé issu de la levure provoque la germination de $40 \%$ à $50 \%$ des graines.

Nous devons désormais travailler à la production de ces levures à plus grande échelle en utilisant de plus grands fermenteurs; nous explorons également comment délivrer ce composé : peut-on utiliser le milieu de culture «brut » des levures, faut-il extraire chimiquement le composé, le purifier et l'administrer? Ou peut-on fournir les levures aux paysans pour qu'ils brassent cette «bière » dans leur exploitation et qu'ils l'épandent? Ce que nous souhaitons à ce stade est que les investisseurs prennent le relais. D'autre part, nous recevons également un financement de la Fondation Bill Gates pour faire des essais en plein champ de cette molécule. L'important est de faire participer les personnes qui ont besoin de cette technologie, du début à la fin du processus. $\diamond$

\section{LIENS D'INTÉRÊT}

L'auteur déclare n'avoir aucun lien d'intérêt concernant les données publiées dans cet article.

\section{RÉFÉRENCES}

1. Gressel J, Hanafi A, Head G, et al. Major heretofore intractable biotic constraints to African food security that may be amenable to novel biotechnological solutions. Crop Prot $2004 ; 23$ : 661-89.

2. Dörr I. How Striga parasitizes its host: a TEM and SEM study. Ann Bot 1997 ; $79: 463-72$.
TIRÉS À PART

T. Bayer

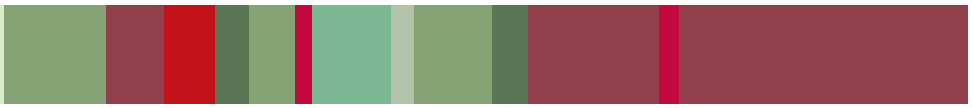

